\title{
Indium-Mediated Reaction of Trialkylsilyl Propargyl Bromide with Aldehydes: Highly \\ Regioselective Synthesis of Allenic and Homopropargylic Alcohols
}

\author{
Man-Jing Lin and Teck-Peng Loh* \\ Department of Chemistry, National University of Singapore, 3 Science Drive 3, \\ Singapore 117543
}

\section{Supporting information:}

\section{Experimental Section}

General: THF was distilled from sodium/benzophenone. Hexane and dichloromethane were distilled from $\mathrm{CaH}_{2}$. TLC was carried out with pre-coated Merck $60 \mathrm{~F}_{254}$ plates. Silica gel 60 (Merck, 400-630 mesh) was used for column chromatography. ${ }^{1} \mathrm{H}$ and ${ }^{13} \mathrm{C}$ NMR spectra were taken in $\mathrm{CDCl}_{3}$ on Bruker DPX300 and referenced to internal tetramethylsilane. MS (EI) spectra were recorded on a Hewlett-Packard 5890A gas chromatrogram. IR spectra were recorded on a Bio-RAD FTS 165 FT-IR Spectrometer.

\section{Indium-Mediated Propargylation of Aldehydes; General Procedure:}

Trimethylsilyl propargyl bromide (191 mg, $1.0 \mathrm{mmol}$, 2 equiv) was added to a mixture of the indium powder (114 mg, $1.0 \mathrm{mmol}, 2$ equiv) and indium tribromide (18 $\mathrm{mg}, 0.05$ mmol, 0.1 equiv) in $1 \mathrm{~mL}$ anhydrous THF at room temperature with stirring under an atmosphere of dry nitrogen. After $15 \mathrm{~min}$, nonyl aldehyde (86 $\mu \mathrm{L}, 0.5 \mathrm{mmol}$, 1 equiv) was added at room temperature. The mixture was refluxed overnight, and finally quenched with $10 \mathrm{~mL}$ of $1 \mathrm{M} \mathrm{HCl}$ solution. The aqueous layer was extracted with ethyl acetate $(10 \mathrm{~mL} \times 3)$. The combined organic extracts were washed with brine, dried over anhydrous magnesium sulfate, concentrated under vacuum, and purified by flash silica gel column chromatography to provide $121 \mathrm{mg}$ (95\% yield) of 1-Trimethylsilanyl-dodec1-yn-4-ol (5e) as a clear oil.

\section{1-Phenyl-6-trimethylsilanyl-hex-5-yn-3-ol (5a)}<smiles>N#CC#CCC(O)CCc1ccccc1</smiles>

Yield\%: 85\%

$\mathrm{R}_{\mathrm{f}} 0.40$ (hexane : ethyl acetate 4:1) 


\section{${ }^{1}$ H NMR: $\quad\left(300 \mathrm{MHz}, \mathrm{CDCl}_{3}\right)$}

$\delta$ 7.31-7.26 (m, 2H, phenyl), 7.22-7.20 (m, 3H, phenyl), 3.76 (m, 1H, CHOH), 2.73 (m, 2H, $\mathrm{PhCH}_{2}$ ), 2.48 (dd, $J=4.86,16.71 \mathrm{~Hz}, 1 \mathrm{H}, \mathrm{C} \equiv \mathrm{CCHH}$ ), 2.38 (dd, $J=6.63,16.71 \mathrm{~Hz}$, $1 \mathrm{H}, \mathrm{C} \equiv \mathrm{CCHH}), 1.85$ (m, 2H, $\left.\mathrm{PhCH}_{2} \mathrm{CH}_{2}\right), 0.16$ (s, 9H, Si(CH$\left.)_{3}\right) \mathrm{ppm}$

\section{${ }^{13}$ C NMR: $\quad\left(75 \mathrm{MHz}, \mathrm{CDCl}_{3}\right)$}

$\delta$ 141.7, 128.5, 128.4, 125.9, $103.0(\mathbf{C} \equiv \mathrm{CSi}), 87.8(\mathbf{C H O H}), 69.1(\mathrm{C} \equiv \mathbf{C S i}), 37.8$ $\left(\mathrm{CH}_{2} \mathrm{CHOH}\right), 31.8\left(\mathrm{PhCH}_{2}\right), 29.0\left(\mathrm{CH}_{2} \mathrm{C} \equiv \mathrm{C}\right), 0.11\left(\mathrm{Si}\left(\mathrm{CH}_{3}\right)_{3}\right) \mathrm{ppm}$

FTIR: $\quad 3434,3020,2961,2170,1718,1492,1454,1411,1251,1216,1040,845$, 758, $700,669 \mathrm{~cm}^{-1}$

EIHRMS: $\quad$ Calcd for $\mathrm{C}_{15} \mathrm{H}_{22} \mathrm{OSi}$ : 246.1440, found : 246.1396 .

\section{1-Phenyl-6-trimethylsilanyl-hex-1-en-5-yn-3-ol (5b)}<smiles>OC(/C=C/c1ccccc1)CC#CCS</smiles>

Yield\%: 89\%

$\mathrm{R}_{\mathrm{f}} 0.40$ (hexane : ethyl acetate $4: 1$ )

${ }^{1}$ H NMR: $\quad\left(300 \mathrm{MHz}, \mathrm{CDCl}_{3}\right)$

$\delta$ 7.41-7.25 (m, 5H, Phenyl), 6.67 (d, $J=16.0$ Hz, 1H, PhCH), 6.27 (dd, $J=5.91,9.75$ $\mathrm{Hz}, 1 \mathrm{H}, \mathrm{PhCH}=\mathrm{CH}), 4.45$ (m, 1H, CHOH), 2.62 (dd, $J=4.5,16.71 \mathrm{~Hz}, 1 \mathrm{H}, \mathrm{C} \equiv \mathrm{CCHH})$, 2.36 (dd, $J=6.27,16.71 \mathrm{~Hz}, 1 \mathrm{H}, \mathrm{C} \equiv \mathrm{CCHH}), 2.25$ (br, 1H, OH), 0.176 (s, 9H, Si(CH$\left.)_{3}\right)$ ppm

13 C NMR: $\quad\left(75 \mathrm{MHz}, \mathrm{CDCl}_{3}\right)$

$\delta$ 136.5, 131.1, 130.2, 128.6, 127.8 (PhCH), 126.6 (PhCH=CH), $102.5(\mathbf{C} \equiv \mathrm{CSi}), 88.1$ $(\mathbf{C H O H}), 70.7(\mathrm{C} \equiv \mathbf{C S i}), 29.3\left(\mathbf{C H}_{2} \mathrm{CHOH}\right), 0.06\left(\mathrm{Si}\left(\mathbf{C H}_{3}\right)_{3}\right) \mathrm{ppm}$

FTIR: $\quad 3366,3027,2958,2900,2358,2176,1933,1600,1493,1449,1250,1100$, 1029, 966, 843, 748, $693 \mathrm{~cm}^{-1}$ 
EIHRMS: $\quad$ Calcd for $\mathrm{C}_{15} \mathrm{H}_{20} \mathrm{OSi}: 244.1283$, found : 244.1278 .

\section{1-Cyclohexyl-4-trimenthylsilanyl-but-3-yn-ol (5c)}<smiles>CC(C)(C)C#CCC(O)C1CCCCC1</smiles>

Yield\%: 90\%

$\mathrm{R}_{\mathrm{f}} 0.48$ (hexane : ethyl acetate $4: 1$ )

${ }^{1}$ H NMR: $\quad\left(300 \mathrm{MHz}, \mathrm{CDCl}_{3}\right)$

$\delta 3.43$ (m, 1H, CHOH), 2.43 (dd, $J=3.6,16.71 \mathrm{~Hz}, 1 \mathrm{H}, \mathrm{C} \equiv \mathrm{CCHH}$ ), 2.32 (dd, $J=7.65$, $16.71 \mathrm{~Hz}, 1 \mathrm{H}, \mathrm{C} \equiv \mathrm{CCHH}), 2.12$ (br, 1H, OH), 1.21-0.96 (m, 11H, c- $\mathrm{C}_{6} \mathbf{H}_{11}$ ), 0.117 (s, 9H, $\left.\mathrm{Si}\left(\mathrm{CH}_{3}\right)_{3}\right) \mathrm{ppm}$

${ }^{13}$ C NMR: $\quad\left(75 \mathrm{MHz}, \mathrm{CDCl}_{3}\right)$

$\delta \quad 103.8 \quad(\mathrm{C} \equiv \mathrm{CSi}), \quad 87.3 \quad(\mathrm{CHOH}), \quad 73.8 \quad(\mathrm{C} \equiv \mathbf{C S i}), \quad 42.6 \quad(\mathrm{CHCHOH}), \quad 28.9, \quad 28.1$ ( $\left.\mathrm{CH}_{2} \mathrm{CHOH}\right)$, 26.4, 26.1, 26.0 (2C, overlap), $0.01\left(\mathrm{Si}\left(\mathrm{CH}_{3}\right)_{3}\right) \mathrm{ppm}$

FTIR: $\quad 3402,2928,2851,2663,2174,1713,1450,1422,1349,1249,1198,1103$, 1086, 1015, 842, 760, 698, $651 \mathrm{~cm}^{-1}$

EIHRMS: $\quad$ Calcd for $\mathrm{C}_{13} \mathrm{H}_{24} \mathrm{OSi}: 224.1596$, found : 224.1594 .

1-Phenyl-4-trimethylsilanyl-but-3-yn-1-ol (5d)

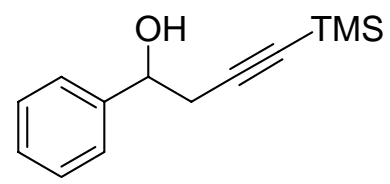

Yield\%: 92\%

$\mathrm{R}_{\mathrm{f}} 0.38$ (hexane : ethyl acetate $4: 1$ )

${ }^{1}$ H NMR: $\quad\left(300 \mathrm{MHz}, \mathrm{CDCl}_{3}\right)$ 
$\delta$ 7.38-7.26 (m, 5H, phenyl), 4.84 (t, $J=6.27 \mathrm{~Hz}, 1 \mathrm{H}, \mathrm{PhCHOH}), 2.65$ (d, $J=6.27 \mathrm{~Hz}$, $2 \mathrm{H}, \mathrm{CH}_{2} \mathrm{C} \equiv \mathrm{CSi}$ ), 2.45 (brd, $1 \mathrm{H}, \mathrm{CHOH}$ ), 0.17(s, 9H, $\left.\mathrm{Si}\left(\mathrm{CH}_{3}\right)_{3}\right) \mathrm{ppm}$

\section{${ }^{13}$ C NMR: $\quad\left(75 \mathrm{MHz}, \mathrm{CDCl}_{3}\right)$}

8142.5, 128.9, 127.8, 125.7, 103.0, $87.8\left(\mathrm{CH}_{2} \mathbf{C} \equiv \mathrm{CSi}\right), 72.3(\mathrm{CHOH}), 31.0\left(\mathrm{CHCH}_{2} \mathrm{C}\right)$, $0.06\left(\mathrm{Si}\left(\mathrm{CH}_{3}\right)_{3}\right) \mathrm{ppm}$

FTIR: $\quad$ (neat) v 3396, 3033, 2960, 2902, 2177, 1724, 1604, 1494, 1454, 1250, $1202,1039,846,759,700 \mathrm{~cm}^{-1}$

EIHRMS: $\quad$ Calcd for $\mathrm{C}_{13} \mathrm{H}_{18} \mathrm{OSi}: 218.1127$, found: 218.1125

\section{1-Trimethylsilanyl-dodec-1-yn-4-ol (5e)}

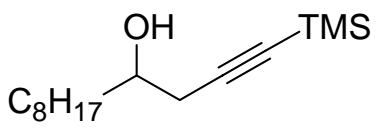

Yield\%: 95\%

$\mathrm{R}_{\mathrm{f}} 0.45$ (hexane : ethyl acetate $4: 1$ )

${ }^{1}$ H NMR: $\quad\left(300 \mathrm{MHz}, \mathrm{CDCl}_{3}\right)$

$\delta 3.71$ (m, 1H, CHOH), 2.45 (dd, $J=4.89,16.71 \mathrm{~Hz}, 1 \mathrm{H}, \mathrm{C} \equiv \mathrm{CCHH}$ ), 2.33 (dd, $J=6.96$, $16.71 \mathrm{~Hz}, 1 \mathrm{H}, \mathrm{C} \equiv \mathrm{CCHH}), 1.96$ (br, 1H, OH), 1.50 (m, 2H, $\left.\mathrm{CH}_{2} \mathrm{CHOH}\right), 1.27$ (m, 12H, $\left.\left(\mathrm{CH}_{2}\right)_{6}\right), 0.87$ (t, $\left.J=5.9 \mathrm{~Hz}, 3 \mathrm{H}, \mathrm{CH}_{3}\right), 0.15$ (s, 9H, $\left.\mathrm{Si}\left(\mathrm{CH}_{3}\right)_{3}\right) \mathrm{ppm}$

${ }^{13}$ C NMR: $\quad\left(75 \mathrm{MHz}, \mathrm{CDCl}_{3}\right)$

$\delta 103.4(\mathbf{C} \equiv \mathrm{CSi}), 86.9(\mathbf{C H O H}), 70.0(\mathrm{C} \equiv \mathbf{C S i}), 36.2\left(\mathrm{CH}_{2} \mathrm{CHOH}\right), 31.9,29.5,29.5,29.3$, 28.9, 25.6, $22.7\left(\mathrm{CH}_{2} \mathrm{CH}_{3}\right), 14.1\left(\mathrm{CH}_{3}\right), 0.07\left(\mathrm{Si}\left(\mathbf{C H}_{3}\right)_{3}\right) \mathrm{ppm}$

FTIR: $\quad 3365,2957,2926,2855,2176,1465,1250,1084,1005,842,760,698,648$ $\mathrm{cm}^{-1}$

EIHRMS: $\quad$ Calcd for $\mathrm{C}_{15} \mathrm{H}_{30} \mathrm{OSi}: 254.2066$, found : 254.2067.

\section{1-Triisopropylsilanyl-dodec-1-yn-4-ol (9a)}


CliPS

Yield\%: 58\%

$\mathrm{R}_{\mathrm{f}} 0.52$ (hexane : ethyl acetate $4: 1$ )

${ }^{1} \mathrm{H}$ NMR: $\quad\left(300 \mathrm{MHz}, \mathrm{CDCl}_{3}\right)$

$\delta 3.72$ (m, 1H, CHOH), 2.50 (dd, $J=4.86,16.71 \mathrm{~Hz}, 1 \mathrm{H}, \mathrm{C} \equiv \mathrm{CCHH}), 2.39$ (dd, $J=6.6$, $16.71 \mathrm{~Hz}, 1 \mathrm{H}, \mathrm{C} \equiv \mathrm{CCHH}), 1.27$ (m, 14H, $\left.\left(\mathrm{CH}_{2}\right)_{7}\right), 1.07$ (m, 21H, Si $\left.\left(\mathrm{CH}\left(\mathrm{CH}_{3}\right)_{2}\right)_{3}\right), 0.88$ (t, $\left.J=6.96 \mathrm{~Hz}, 3 \mathrm{H}, \mathrm{CH}_{3}\right) \mathrm{ppm}$

13 C NMR: $\quad\left(75 \mathrm{MHz}, \mathrm{CDCl}_{3}\right)$

$\delta 104.8(\mathbf{C} \equiv \mathrm{CSi}), 83.6(\mathbf{C H O H}), 70.1(\mathrm{C} \equiv \mathbf{C S i}), 36.2,31.9,29.6,29.5,29.3,28.9$ $\left(\mathbf{C H}_{2} \mathrm{C} \equiv \mathrm{C}\right), 25.6,22.7,18.6\left(\mathrm{SiCH}\left(\mathrm{CH}_{3}\right)_{2}\right), 14.1\left(\mathrm{CH}_{3}\right), 11.2\left(\mathrm{SiCH}\left(\mathrm{CH}_{3}\right)_{2}\right) \mathrm{ppm}$

FTIR: $\quad 3429,2927,2856,2177,1712,1648,1466,1378,1250,1033,843,760$ $\mathrm{cm}^{-1}$.

EIHRMS: $\quad$ Calcd for $\mathrm{C}_{21} \mathrm{H}_{42} \mathrm{OSi}: 338.3055$, found : 338.3017.

1-(tert-Butyl-diphenyl-silanyl)-dodec-1-yn-4-ol (9f)

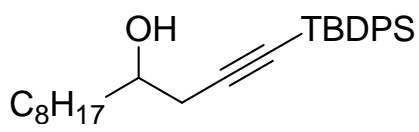

Yield\%: 35\%

$\mathrm{R}_{\mathrm{f}} 0.49$ (hexane : ethyl acetate $4: 1$ )

${ }^{1} \mathrm{H}$ NMR: $\quad\left(300 \mathrm{MHz}, \mathrm{CDCl}_{3}\right)$

$\delta 7.79$ (m, 4H, Phenyl), 7.37 (m, 6H, Phenyl), 3.85 (m, 1H, CHOH), 2.65 (dd, $J=5.22$, $17.07 \mathrm{~Hz}, 1 \mathrm{H}, \mathrm{C} \equiv \mathrm{CCHH}$ ), 2.55 (dd, $J=6.6,17.07 \mathrm{~Hz}, 1 \mathrm{H}, \mathrm{C} \equiv \mathrm{CCHH}), 1.28$ (m, 14H, $\left.\left(\mathrm{CH}_{2}\right)_{7}\right), 1.09$ (s, 9H, $\mathrm{Si}\left(\mathrm{C}\left(\mathrm{CH}_{3}\right)_{3}\right), 0.88$ (t, $\left.J=6.96 \mathrm{~Hz}, 3 \mathrm{H}, \mathrm{CH}_{3}\right) \mathrm{ppm}$

${ }^{13}$ C NMR: $\quad\left(75 \mathrm{MHz}, \mathrm{CDCl}_{3}\right)$ 
$\delta$ 135.5, 135.5, 133.5, 133.5, 129.5, 129.5, 127.7, 127.7, 107.6 (C $\equiv \mathrm{CSi}), 82.8(\mathbf{C H O H})$, 70.2 (C三CSi), 36.4, 31.9, 29.6, 29.5, 29.3, $29.2\left(\mathrm{CH}_{2} \mathrm{C} \equiv \mathrm{C}\right), 27.1\left(\mathrm{SiC}\left(\mathrm{CH}_{3}\right)_{3}\right)$, 25.6, 22.7, $18.5\left(\mathrm{SiC}\left(\mathrm{CH}_{3}\right)_{3}\right), 14.1\left(\mathrm{CH}_{3}\right) \mathrm{ppm}$

FTIR: $\quad 3359,2959,2928,2856,2175,1428,1378,1109,820 \mathrm{~cm}^{-1}$.

ESIHRMS: $\quad$ Calcd for $\mathrm{C}_{28} \mathrm{H}_{40} \mathrm{OSiNa}: 443.2746$, found : 443.2751 .

\section{0-(1'-Hydroxy-4' -Trimethylsilanyl-3'-butyn-1'-yl)-3-oxopregn-4-ene (5f)}

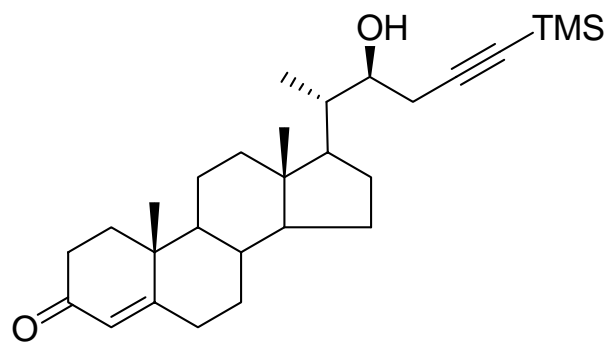

Yield\%: 65\%

$\mathrm{R}_{\mathrm{f}} 0.50$ (hexane : ethyl acetate $1: 1$ )

${ }^{1}$ H NMR: $\quad\left(300 \mathrm{MHz}, \mathrm{CDCl}_{3}\right)$

$\delta 5.72$ (s, 1H, 4-CH), 3.87 (m, 1H, 22-CH), 1.18 (s, 3H, 19-CH ${ }_{3}$ ), 0.91 (d, $J=6.63 \mathrm{~Hz}$, $\left.3 \mathrm{H}, 21-\mathrm{CH}_{3}\right), 0.73$ (s, 3H, 18-CH 3$), 0.16\left(\mathrm{~s}, 9 \mathrm{H}, \mathrm{Si}\left(\mathrm{CH}_{3}\right)_{3}\right) \mathrm{ppm}$

${ }^{13} \mathrm{C}$ NMR: $\quad\left(75 \mathrm{MHz}, \mathrm{CDCl}_{3}\right)$

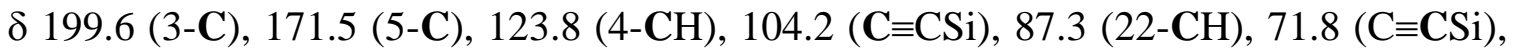
55.8, 53.8, 52.6, 42.4, 39.6, 39.4, 38.6, 35.7, 34.0, 32.9, 32.0, 29.7, 27.7, 26.9, 24.1, 21.1, 17.4, 15.3, 11.8, 11.5, $0.08\left(\mathrm{Si}\left(\mathrm{CH}_{3}\right)_{3}\right) \mathrm{ppm}$

FTIR: $\quad 3445,3019,2937,1659,1455,1215,1024,844,756,669 \mathrm{~cm}^{-1}$

EIHRMS: $\quad$ Calcd for $\mathrm{C}_{28} \mathrm{H}_{44} \mathrm{O}_{2} \mathrm{Si}: 440.3111$, found : 440.3102

20-(1'-Hydroxy-3'-butyn-1'-yl)-3-oxopregn-4-ene (10) 


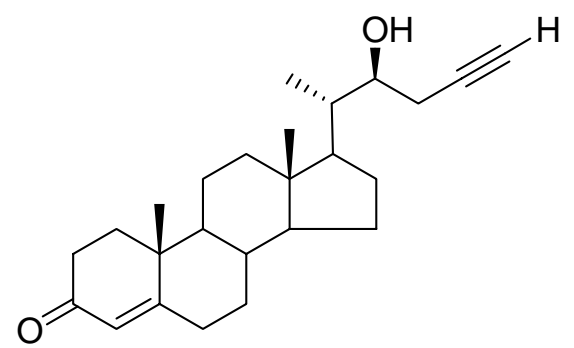

Yield\%: 90\%

$\mathrm{R}_{\mathrm{f}} 0.41$ (hexane : ethyl acetate $1: 1$ )

${ }^{1}$ H NMR: $\quad\left(300 \mathrm{MHz}, \mathrm{CDCl}_{3}\right)$

$\delta 5.73$ (s, 1H, 4-CH), 3.90 (m, 1H, 22-CH), 1.19 (s, 3H, 19-CH CH $_{3} 0.92$ (d, $J=6.63 \mathrm{~Hz}$, $\left.3 \mathrm{H}, 21-\mathrm{CH}_{3}\right), 0.73$ (s, 3H, 18-CH 3 ) ppm

${ }^{13} \mathrm{C}$ NMR: $\quad\left(75 \mathrm{MHz}, \mathrm{CDCl}_{3}\right)$

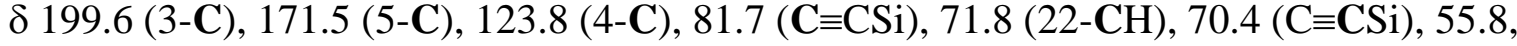
53.8, 52.5, 42.4, 39.7, 39.6, 38.6, 35.7, 34.0, 33.0, 32.0, 29.7, 27.7, 25.5, 24.1, 21.1, 17.4, $11.9,11.4 \mathrm{ppm}$

FTIR: $\quad 3435,3017,2941,2360,1659,1444,1216,756,668 \mathrm{~cm}^{-1}$

EIHRMS: $\quad$ Calcd for $\mathrm{C}_{25} \mathrm{H}_{36} \mathrm{O}_{2}: 368.2715$, found : 368.2713 .

20-(1-Hydroxy-3-buten1-yl)-3-oxopregn-4-ene (11)

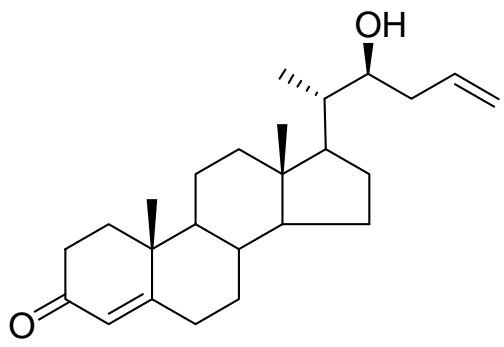

Yield\%: 98\%

$\mathrm{R}_{\mathrm{f}} 0.51$ (hexane : ethyl acetate 2:1)

${ }^{1}$ H NMR: $\quad\left(300 \mathrm{MHz}, \mathrm{CDCl}_{3}\right)$ 
$\delta$ 5.87-5.76 (m, 1H, CH=CH $), 5.73(\mathrm{br}, 1 \mathrm{H}, 4-\mathrm{CH}), 5.13\left(\mathrm{~d}, J=7.65 \mathrm{~Hz}, 1 \mathrm{H}, \mathrm{CH}=\mathrm{CH}_{2}\right.$ ), 5.09 (br, $1 \mathrm{H}, \mathrm{CH}=\mathrm{CH}_{2}$ ), 3.71 (dd, $\left.J=4.89,8.36 \mathrm{~Hz}, 1 \mathrm{H}, 22-\mathrm{CH}\right), 1.18$ (s, 3H, 19-CH ), $^{2}$ 0.93 (d, $\left.J=5.91 \mathrm{~Hz}, 3 \mathrm{H}, 21-\mathrm{CH}_{3}\right), 0.72\left(\mathrm{~s}, 3 \mathrm{H}, 18-\mathrm{CH}_{3}\right) \mathrm{ppm}$

\section{${ }^{13} \mathrm{C}$ NMR: $\quad\left(75 \mathrm{MHz}, \mathrm{CDCl}_{3}\right)$}

199.6 (3-C), 171.5 (5-C), $135.7\left(\mathrm{CH}_{2}=\mathbf{C H}\right), 123.8(4-\mathbf{C H}), 117.5\left(\mathbf{C H}_{2}=\mathrm{CH}\right), 72.4$ (22CH), 55.8, 53.8, 52.6, 42.4, 40.2, 40.1, 39.7, 38.6, 35.7 (2C, overlap), 34.0, 32.9, 32.0, 27.7, 24.1, 21.1, 17.4, 11.9, $11.7 \mathrm{ppm}$

FTIR: $\quad 3368,2939,1674,1432,1229,886 \mathrm{~cm}^{-1}$.

EIHRMS: $\quad$ Calcd for $\mathrm{C}_{25} \mathrm{H}_{38} \mathrm{O}_{2}$ : 370.2888, found : 370.2872 .

\section{Indium-Mediated Allenylation of Aldehydes; General Procedure:}

To a suspension of indium powder (57 mg, $0.5 \mathrm{mmol}$, 2 equiv) in $\mathrm{H}_{2} \mathrm{O} / \mathrm{THF}$ (0.5 mL, 5:1) was added nonyl aldehyde ( $43 \mu \mathrm{L}, 0.25 \mathrm{mmol}, 1$ equiv) and then triisopropylsilyl propargyl bromide (69 mg, 2 equiv). The mixture was vigorously stirred at room temperature for 10 hours. Standard workup and purified by flash silica gel column chromatography to provide $44 \mathrm{mg}$ (52\% yield) 3-Triisopropylsilanyl-dodeca-1,2-dien-4ol (8a).

\section{3-Triisopropylsilanyl-dodeca-1,2-dien-4-ol (8a)}

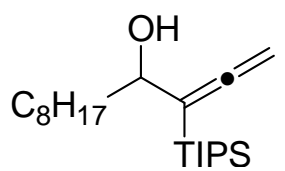

Yield\%: 52\%

$\mathrm{R}_{\mathrm{f}} 0.55$ (hexane : ethyl acetate $4: 1$ )

${ }^{1}$ H NMR: $\quad\left(300 \mathrm{MHz}, \mathrm{CDCl}_{3}\right)$

$\delta 4.56\left(\mathrm{~m}, 2 \mathrm{H}, \mathrm{C}=\mathrm{C}=\mathrm{CH}_{2}\right), 4.01(\mathrm{~m}, 1 \mathrm{H}, \mathrm{CHOH}), 1.27\left(\mathrm{~m}, 14 \mathrm{H},\left(\mathrm{CH}_{2}\right)_{7}\right), 1.09(\mathrm{~m}, 21 \mathrm{H}$, $\left.\mathrm{Si}\left(\mathrm{CH}\left(\mathrm{CH}_{3}\right)_{2}\right)_{3}\right), .088\left(\mathrm{t}, J=6.45 \mathrm{~Hz}, 3 \mathrm{H}, \mathrm{CH}_{3}\right) \mathrm{ppm}$

${ }^{13}$ C NMR: $\quad\left(75 \mathrm{MHz}, \mathrm{CDCl}_{3}\right)$

$\delta$ 209.6 $\left(\mathrm{C}=\mathbf{C}=\mathrm{CH}_{2}\right), 96.9\left(\mathbf{C}=\mathrm{C}=\mathrm{CH}_{2}\right), 72.4(\mathbf{C H O H}), 69.8\left(\mathrm{C}=\mathrm{C}=\mathrm{CH}_{2}\right)$, 38.3, 31.9, 29.6, 29.5, 29.3, 26.2, 22.7, $\left.18.7\left(\mathrm{CH}\left(\mathrm{CH}_{3}\right)_{2}\right), 14.1\left(\mathrm{CH}_{3}\right), 11.7\left(\mathrm{SiCH}\left(\mathrm{CH}_{3}\right)_{2}\right)_{3}\right) \mathrm{ppm}$ 
FTIR: $\quad 3468,2927,2866,1922,1465,1067,1008,883,811,761 \mathrm{~cm}^{-1}$.

EIHRMS: $\quad$ Calcd for $\mathrm{C}_{21} \mathrm{H}_{42} \mathrm{OSi}: 338.3005$, found : 338.3004 .

\section{1-Phenyl-4-triisopropylsilanyl-hexa-4,5-dien-3-ol (8b)}<smiles>C=CC(O)C(CCc1ccccc1)C(=O)I</smiles>

Yield\%: 71\%

$\mathrm{R}_{\mathrm{f}} 0.54$ (hexane : ethyl acetate 4:1)

${ }^{1}$ H NMR: $\quad\left(300 \mathrm{MHz}, \mathrm{CDCl}_{3}\right)$

$\delta$ 7.32-7.16 (m, 5H, Phenyl), 4.61 (m, $2 \mathrm{H}, \mathrm{C}=\mathrm{C}=\mathrm{CH}_{2}$ ), 4.06 (brs, $\left.1 \mathrm{H}, \mathrm{CHOH}\right), 2.78$ (m, $\left.2 \mathrm{H}, \mathrm{CH}_{2} \mathrm{Ar}\right), 1.90$ (m, 2H, $\left.\mathrm{CH}_{2} \mathrm{CH}_{2} \mathrm{Ar}\right), 1.08\left(\mathrm{~m}, 21 \mathrm{H}, \mathrm{Si}\left(\mathrm{CH}\left(\mathrm{CH}_{3}\right)_{2}\right)_{3}\right) \mathrm{ppm}$

${ }^{13}$ C NMR: $\quad\left(75 \mathrm{MHz}, \mathrm{CDCl}_{3}\right)$

$\delta 209.6\left(\mathrm{C}=\mathbf{C}=\mathrm{CH}_{2}\right), 142.1,128.5,128.3,125.8,96.7\left(\mathbf{C}=\mathrm{C}=\mathrm{CH}_{2}\right), 72.6(\mathbf{C H O H}), 69.1$ $\left.\left(\mathrm{C}=\mathrm{C}=\mathrm{CH}_{2}\right), 40.1\left(\mathrm{CH}_{2} \mathrm{CH}_{2} \mathrm{Ar}\right), 32.6\left(\mathrm{CH}_{2} \mathrm{Ar}\right), 18.6\left(\mathrm{CH}_{3}\right), 11.6\left(\mathrm{SiCH}\left(\mathrm{CH}_{3}\right)_{2}\right)_{3}\right) \mathrm{ppm}$

FTIR: $\quad 3449,2944,2866,1922,1717,1450,883,809,748,699 \mathrm{~cm}^{-1}$.

EIHRMS: $\quad$ Calcd for $\mathrm{C}_{21} \mathrm{H}_{34} \mathrm{OSi}: 330.2379$, found : 330.2390 .

1-Phenyl-4-triisopropylsilanyl-hexa-1,4,5-trien-3-ol (8c)<smiles>C=CC(O)C(=CC=Cc1ccccc1)C(F)(F)F</smiles>

Yield\%: 46\%

$\mathrm{R}_{\mathrm{f}} 0.54$ (hexane : ethyl acetate $4: 1$ )

${ }^{1}$ H NMR: $\quad\left(300 \mathrm{MHz}, \mathrm{CDCl}_{3}\right)$ 
$\delta$ 7.40-7.21 (m, 5H, Phenyl), 6.58 (d, $J=16.02 \mathrm{~Hz}, 1 \mathrm{H}, \mathrm{PhCH}=\mathrm{CH}), 6.30$ (dd, $J=6.69$, $15.69 \mathrm{~Hz}, 1 \mathrm{H}, \mathrm{PhCH}=\mathrm{CH}), 4.71(\mathrm{~m}, 1 \mathrm{H}, \mathrm{CHOH}), 4.66\left(\mathrm{~m}, 2 \mathrm{H}, \mathrm{C}=\mathrm{C}=\mathrm{CH} \mathbf{H}_{2}\right), 1.08(\mathrm{~m}, 21 \mathrm{H}$, $\left.\operatorname{SiCH}\left(\mathrm{CH}_{3}\right)_{3}\right) \mathrm{ppm}$

13 C NMR: $\quad\left(75 \mathrm{MHz}, \mathrm{CDCl}_{3}\right)$

$\delta 210.1\left(\mathrm{C}=\mathbf{C}=\mathrm{CH}_{2}\right), 131.8,130.3,129.7,128.6,127.7,126.6,96.3\left(\mathbf{C}=\mathrm{C}=\mathrm{CH}_{2}\right), 73.0$ $\left.(\mathbf{C H O H}), 70.9\left(\mathrm{C}=\mathrm{C}=\mathbf{C H}_{2}\right), 18.7\left(\mathrm{CH}_{3}\right), 11.7\left(\mathrm{SiCH}\left(\mathrm{CH}_{3}\right)_{2}\right)_{3}\right) \mathrm{ppm}$

FTIR: $\quad 3626,3020,2394,1657,1218,925,776,743 \mathrm{~cm}^{-1}$.

EIHRMS: $\quad$ Calcd for $\mathrm{C}_{21} \mathrm{H}_{32} \mathrm{OSi}$ : 328.2222, found : 328.2213.

\section{1-Cyclohexyl-2-triiopropylsilanyl-buta-2,3-dien-1-ol (8d)}<smiles>C=C=C([AlH2])C(O)C1CCCCC1</smiles>

Yield\%: 61\%

$\mathrm{R}_{\mathrm{f}} 0.52$ (hexane : ethyl acetate $4: 1$ )

${ }^{1} \mathrm{H}$ NMR: $\quad\left(300 \mathrm{MHz}, \mathrm{CDCl}_{3}\right)$

$\delta 4.55\left(\mathrm{~m}, 2 \mathrm{H}, \mathrm{C}=\mathrm{C}=\mathrm{CH}_{2}\right), 3.83(\mathrm{t}, J=5.58 \mathrm{~Hz}, 1 \mathrm{H}, \mathrm{CHOH}), 1.27-0.92(\mathrm{~m}, 11 \mathrm{H}, \mathrm{c}-$ $\left.\mathrm{C}_{6} \mathbf{H}_{11}\right), 1.09\left(\mathrm{~m}, 21 \mathrm{H}, \mathrm{Si}\left(\mathrm{CH}\left(\mathrm{CH}_{3}\right)_{2}\right)_{3}\right) \mathrm{ppm}$

13 C NMR: $\quad\left(75 \mathrm{MHz}, \mathrm{CDCl}_{3}\right)$

$\delta \quad 209.4 \quad\left(\mathrm{C}=\mathbf{C}=\mathrm{CH}_{2}\right), \quad 95.4 \quad\left(\mathbf{C}=\mathrm{C}=\mathrm{CH}_{2}\right), \quad 74.0 \quad(\mathbf{C H O H}), \quad 72.2 \quad\left(\mathrm{C}=\mathrm{C}=\mathrm{CH}_{2}\right), \quad 43.5$ (CHCHOH), 31.1, 26.7, 26.5, 26.5, 26.1, $18.7\left(\mathrm{CH}_{3}\right), 11.8\left(\mathrm{Si}\left(\mathbf{C H}\left(\mathrm{CH}_{3}\right)_{2}\right)_{3}\right) \mathrm{ppm}$

FTIR: $\quad 3429,2930,2866,1928,1637,1459,1217,1077,1002,873,758 \mathrm{~cm}^{-1}$.

EIHRMS: $\quad$ Calcd for $\mathrm{C}_{19} \mathrm{H}_{36} \mathrm{OSi}: 308.2535$, found : 308.2533.

\section{1-Phenyl-2-triisopropylsilanyl-buta-2,3-dien-1ol (8e)}


<smiles>C=C=C([AlH2])C(O)c1ccccc1</smiles>

Yield\%: 59\%

$\mathrm{R}_{\mathrm{f}} 0.55$ (hexane : ethyl acetate $4: 1$ )

${ }^{1} \mathrm{H}$ NMR: $\quad\left(300 \mathrm{MHz}, \mathrm{CDCl}_{3}\right)$

$\delta$ 7.41-7.25 (m, 5H, Phenyl), 5.16 (s, 1H, CHOH), $4.64\left(\mathrm{~m}, 2 \mathrm{H}, \mathrm{C}=\mathrm{C}=\mathrm{CH}_{2}\right), 1.08$ (m, $\left.21 \mathrm{H}, \mathrm{Si}\left(\mathrm{CH}\left(\mathrm{CH}_{3}\right)_{2}\right)_{3}\right) \mathrm{ppm}$

13 C NMR: $\quad\left(75 \mathrm{MHz}, \mathrm{CDCl}_{3}\right)$

$\delta 209.8\left(\mathrm{C}=\mathbf{C}=\mathrm{CH}_{2}\right), 143.4,128.3,127.8,127.2,97.4\left(\mathbf{C}=\mathrm{C}=\mathrm{CH}_{2}\right), 73.2(\mathbf{C H O H}), 72.5$ $\left.\left(\mathrm{C}=\mathrm{C}=\mathrm{CH}_{2}\right), 18.6\left(\mathrm{CH}_{3}\right), 11.7\left(\mathrm{SiCH}\left(\mathrm{CH}_{3}\right)_{2}\right)_{3}\right) \mathrm{ppm}$

FTIR: $\quad 3440,2945,2866,1925,1459,1192,1018,883,817,760,700 \mathrm{~cm}^{-1}$.

EIHRMS: $\quad$ Calcd for $\mathrm{C}_{19} \mathrm{H}_{30} \mathrm{OSi}:$ 302.2066, found : 302.2071 .

3-(tert-Butyl-diphenyl-silanyl)-dodeca-1,2-dien-4-ol (8f)

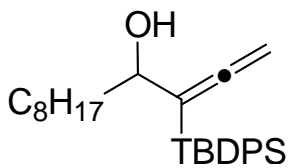

Yield\%: 45\%

$\mathrm{R}_{\mathrm{f}} 0.55$ (hexane : ethyl acetate $4: 1$ )

${ }^{1} \mathrm{H}$ NMR: $\quad\left(300 \mathrm{MHz}, \mathrm{CDCl}_{3}\right)$

$\delta$ 7.72-7.67 (m, 4H, Phenyl), 7.47-7.35 (m, 6H, Phenyl), $4.80\left(\mathrm{~m}, 2 \mathrm{H}, \mathrm{C}=\mathrm{C}=\mathrm{CH}_{2}\right), 3.83$ (br s, 1H, CHOH), 1.38-.098 (m, 14H, $\left.\left(\mathrm{CH}_{2}\right)_{7}\right), 1.14$ (s, 9H, $\left.\mathrm{SiC}\left(\mathrm{CH}_{3}\right)_{3}\right), 0.87$ (t, $J=6.96$ $\left.\mathrm{Hz}, 3 \mathrm{H}, \mathrm{CH}_{3}\right) \mathrm{ppm}$

13 C NMR: $\quad\left(75 \mathrm{MHz}, \mathrm{CDCl}_{3}\right)$ 
$\delta 210.0\left(\mathrm{C}=\mathrm{C}=\mathrm{CH}_{2}\right), 136.3,136.3,133.7 ， 133.7 ， 129.5 ， 129.5 ， 127.8 ， 127.7,96.1$ $\left(\mathbf{C}=\mathrm{C}=\mathrm{CH}_{2}\right), \quad 74.1 \quad(\mathrm{CHOH}), \quad 70.3 \quad\left(\mathrm{C}=\mathrm{C}=\mathrm{CH}_{2}\right), \quad 37.5, \quad 31.8, \quad 29.4, \quad 29.2, \quad 29.2$, 28.1 $\left(\mathrm{SiC}\left(\mathrm{CH}_{3}\right)_{3}\right)$, 25.8, 22.7, $19.5\left(\mathrm{SiC}\left(\mathrm{CH}_{3}\right)_{3}\right), 14.1\left(\mathrm{CH}_{2} \mathbf{C H}_{3}\right) \mathrm{ppm}$

FTIR: $\quad 3582,3081,2928,2857,1925,1463,1428,110,998,820,758,739,701$ $\mathrm{cm}^{-1}$.

EIHRMS: $\quad$ Calcd for $\mathrm{C}_{28} \mathrm{H}_{40} \mathrm{OSi}: 420.2848$, found : 420.2846 .

\section{4-(tert-Butyl-diphenyl-silanyl)-1-phenyl-hexa-4,5-dien-3-ol (8g)}

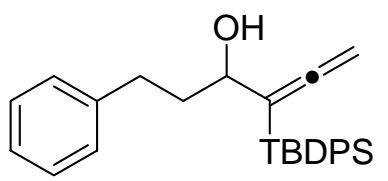

Yield\%: 50\%

$\mathrm{R}_{\mathrm{f}} 0.39$ (hexane : ethyl acetate 4:1)

${ }^{1}$ H NMR: $\quad\left(300 \mathrm{MHz}, \mathrm{CDCl}_{3}\right)$

$\delta$ 7.72-7.67 (m, 4H, Phenyl), 7.44-7.35 (m, 6H, Phenyl), 7.26-7.12 (m, 3H, Phenyl), 6.976.95 (m, 2H, Phenyl), 4.83 (m, 2H, C=C=CH $\mathbf{C H}_{2}$ ), 3.90 (br s, $\left.1 \mathrm{H}, \mathrm{CHOH}\right), 2.67-2.59(\mathrm{~m}$, $1 \mathrm{H}, \mathrm{PhCH}), 2.42-2.30(\mathrm{~m}, 1 \mathrm{H}, \mathrm{PhCH}), 1.70-1.62\left(\mathrm{~m}, 2 \mathrm{H}, \mathrm{PhCH}_{2} \mathrm{CH}_{2}\right), 1.10$ (s, 9H, $\left.\mathrm{SiC}\left(\mathrm{CH}_{3}\right)_{3}\right) \mathrm{ppm}$

${ }^{13}$ C NMR: $\quad\left(75 \mathrm{MHz}, \mathrm{CDCl}_{3}\right)$

$\delta 210.0\left(\mathrm{C}=\mathbf{C}=\mathrm{CH}_{2}\right), 142.0,136.2,136.2,133.6,133.6,129.6,129.5,128.4,128.2,127.9$, 127.8, 125.6, $96.0\left(\mathbf{C}=\mathrm{C}=\mathrm{CH}_{2}\right), 74.3(\mathbf{C H O H}), 69.8\left(\mathrm{C}=\mathrm{C}=\mathrm{CH}_{2}\right), 39.2\left(\mathrm{PhCH}_{2} \mathrm{CH}_{2}\right), 32.4$ $\left(\mathrm{PhCH}_{2}\right) 28.1\left(\mathrm{SiC}\left(\mathrm{CH}_{3}\right)_{3}\right), 19.5\left(\mathrm{SiC}\left(\mathrm{CH}_{3}\right)_{3}\right) \mathrm{ppm}$

FTIR: $\quad 3563,3455,3071,2932,2859,1924,1428,1105,820,757,701,609 \mathrm{~cm}^{-1}$.

EIHRMS: $\quad$ Calcd for $\mathrm{C}_{28} \mathrm{H}_{32} \mathrm{OSi}:$ : 412.2222, found : 412.2236 . 


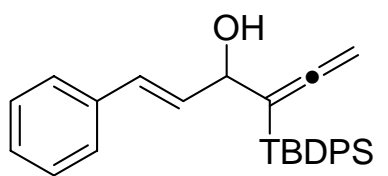

Yield\%: 52\%

$\mathrm{R}_{\mathrm{f}} 0.42$ (hexane : ethyl acetate $4: 1$ )

${ }^{1}$ H NMR: $\quad\left(300 \mathrm{MHz}, \mathrm{CDCl}_{3}\right)$

$\delta$ 7.74-7.68 (m, 4H, Phenyl), 7.46-7.26 (m, 6H, Phenyl), 7.24-7.13 (m, 3H, Phenyl), 7.10 (m, 2H, Phenyl), 6.07-5.96 (m, 2H, PhCH=CH), $4.88(\mathrm{~m}, 2 \mathrm{H}, \mathrm{C}=\mathrm{C}=\mathrm{CH} 2), 4.52$ (br s, $1 \mathrm{H}$, $\mathrm{CHOH}), 1.14$ (s, 9H, $\left.\mathrm{SiC}\left(\mathrm{CH}_{3}\right)_{3}\right) \mathrm{ppm}$

13 C NMR: $\quad\left(75 \mathrm{MHz}, \mathrm{CDCl}_{3}\right)$

$\delta 210.7\left(\mathrm{C}=\mathrm{C}=\mathrm{CH}_{2}\right), 136.8,136.5,136.4,133.5,133.3,130.7,129.9,129.7,129.5,128.3$, 127.8, 127.7, 127.4, 126.5, $95.5\left(\mathbf{C}=\mathrm{C}=\mathrm{CH}_{2}\right), 74.7(\mathbf{C H O H}), 71.4\left(\mathrm{C}=\mathrm{C}=\mathrm{CH}_{2}\right), 28.1$ $\left(\mathrm{SiC}\left(\mathrm{CH}_{3}\right)_{3}\right), 19.5\left(\mathrm{SiC}\left(\mathrm{CH}_{3}\right)_{3}\right) \mathrm{ppm}$

FTIR: $\quad 3583,3226,2931,2861,1629,1106,751,698 \mathrm{~cm}^{-1}$.

EIHRMS: $\quad$ Calcd for $\mathrm{C}_{28} \mathrm{H}_{30} \mathrm{OSi}:$ 410.2066, found : 410.2054 .

2-(tert-Butyl-diphenyl-silanyl)-1-cyclohexyl-buta-2,3-dien-1-ol (8i)<smiles>C=C=C([18OH])C(O)C1CCCCC1</smiles>

Yield\%: 56\%

$\mathrm{R}_{\mathrm{f}} 0.55$ (hexane : ethyl acetate $4: 1$ )

${ }^{1}$ H NMR: $\quad\left(300 \mathrm{MHz}, \mathrm{CDCl}_{3}\right)$

$\delta$ 7.70-7.65 (m, 4H, Phenyl), 7.44-7.33 (m, 6H, Phenyl), 4.79 (m, 2H, C=C=CH $)$, 3.663.63 (m, 1H, CHOH), 1.26-0.88 (m, 11H, c- $\left.\mathrm{C}_{6} \mathbf{H}_{11}\right), 1.14$ (s, 9H, $\left.\mathrm{SiC}\left(\mathrm{CH}_{3}\right)_{3}\right) \mathrm{ppm}$

${ }^{13}$ C NMR: $\quad\left(75 \mathrm{MHz}, \mathrm{CDCl}_{3}\right)$ 
$\delta 209.8\left(\mathrm{C}=\mathrm{C}=\mathrm{CH}_{2}\right), 136.3,136.3 ， 133.8,133.8,129.5 ， 129.5 ， 127.7 ， 127.7,94.8$ $\left(\mathbf{C}=\mathrm{C}=\mathrm{CH}_{2}\right), 74.4(\mathbf{C H O H}), 74.0\left(\mathrm{C}=\mathrm{C}=\mathbf{C H}_{2}\right), 42.6,30.8,28.2\left(\mathrm{SiC}\left(\mathbf{C H}_{3}\right)_{3}\right), 26.4,26.3$, 26.0, 25.7, $19.6\left(\mathrm{SiC}\left(\mathrm{CH}_{3}\right)_{3}\right) \mathrm{ppm}$

FTIR: $\quad 3435,2993,2935,2860,1924,1634,1319,1105,998,832,754,701 \mathrm{~cm}^{-1}$.

EIHRMS: $\quad$ Calcd for $\mathrm{C}_{26} \mathrm{H}_{34} \mathrm{OSi}: 390.2379$, found : 390.2366.

2-(tert-Butyl-diphenyl-silanyl)-1-phenyl-buta-2,3-dien-1-ol (8j)<smiles>C=CC(=O)C(O)c1ccccc1</smiles>

Yield\%: 55\%

$\mathrm{R}_{\mathrm{f}} 0.40$ (hexane : ethyl acetate $4: 1$ )

${ }^{1}$ H NMR: $\quad\left(300 \mathrm{MHz}, \mathrm{CDCl}_{3}\right)$

$\delta$ 7.54-7.51 (m, 2H, Phenyl), 7.42-7.38 (m, 2H, Phenyl), 7.35-7.23 (m, 6H, Phenyl), 7.147.12 (m, 3H, Phenyl), 7.00-6.97 (m, 2H, Phenyl), 4.95 (m, 1H, CHOH), $4.81(\mathrm{~m}, 2 \mathrm{H}$, $\left.\mathrm{C}=\mathrm{C}=\mathrm{CH}_{2}\right), 1.10\left(\mathrm{~s}, 9 \mathrm{H}, \mathrm{SiC}\left(\mathrm{CH}_{3}\right)_{3}\right) \mathrm{ppm}$

${ }^{13}$ C NMR: $\quad\left(75 \mathrm{MHz}, \mathrm{CDCl}_{3}\right)$

$\delta 211.1\left(\mathrm{C}=\mathbf{C}=\mathrm{CH}_{2}\right), 136.3,136.3,133.6,133.6,133.1,129.5,129.5,129.3,127.8,127.8$, 127.6, 127.2, $96.5\left(\mathbf{C}=\mathrm{C}=\mathrm{CH}_{2}\right), 74.5(\mathbf{C H O H}), 73.0\left(\mathrm{C}=\mathrm{C}=\mathbf{C H}_{2}\right), 28.1\left(\mathrm{SiC}\left(\mathbf{C H}_{3}\right)_{3}\right), 19.6$ $\left(\mathrm{SiC}\left(\mathrm{CH}_{3}\right)_{3}\right) \mathrm{ppm}$

FTIR: $\quad 3437,3020,2923,2858,2401,1637,1226,754,669 \mathrm{~cm}^{-1}$.

EIHRMS: $\quad$ Calcd for $\mathrm{C}_{26} \mathrm{H}_{28} \mathrm{OSi}:$ 384.1909, found : 384.1918.

NMR Studies:<smiles>C=CC(=O)C(O)C1CCCCC1C(C)(C)C</smiles> 
Lewis acid: $\mathrm{InBr}_{3}$
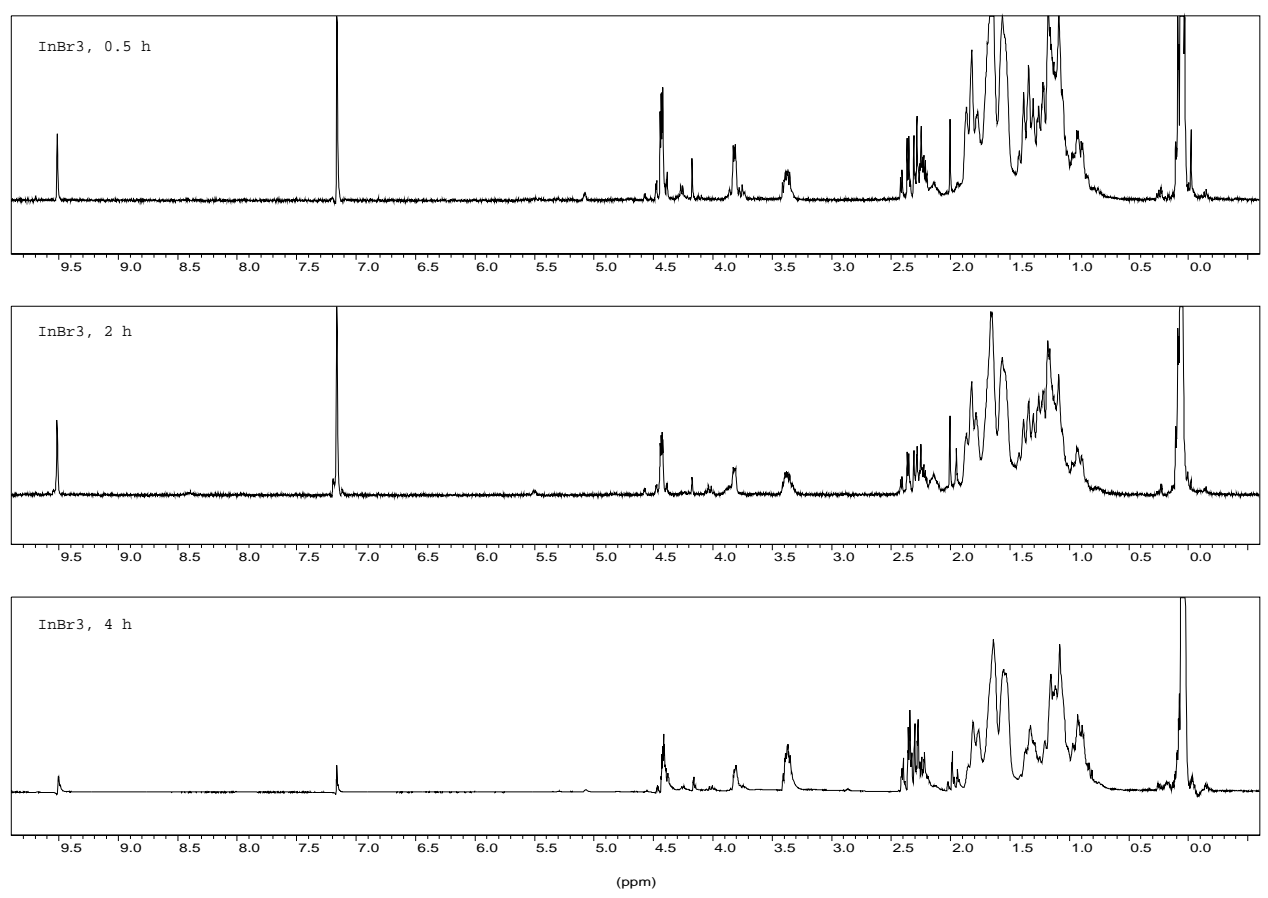

\section{Lewis acid: $\operatorname{InF}_{3}$}
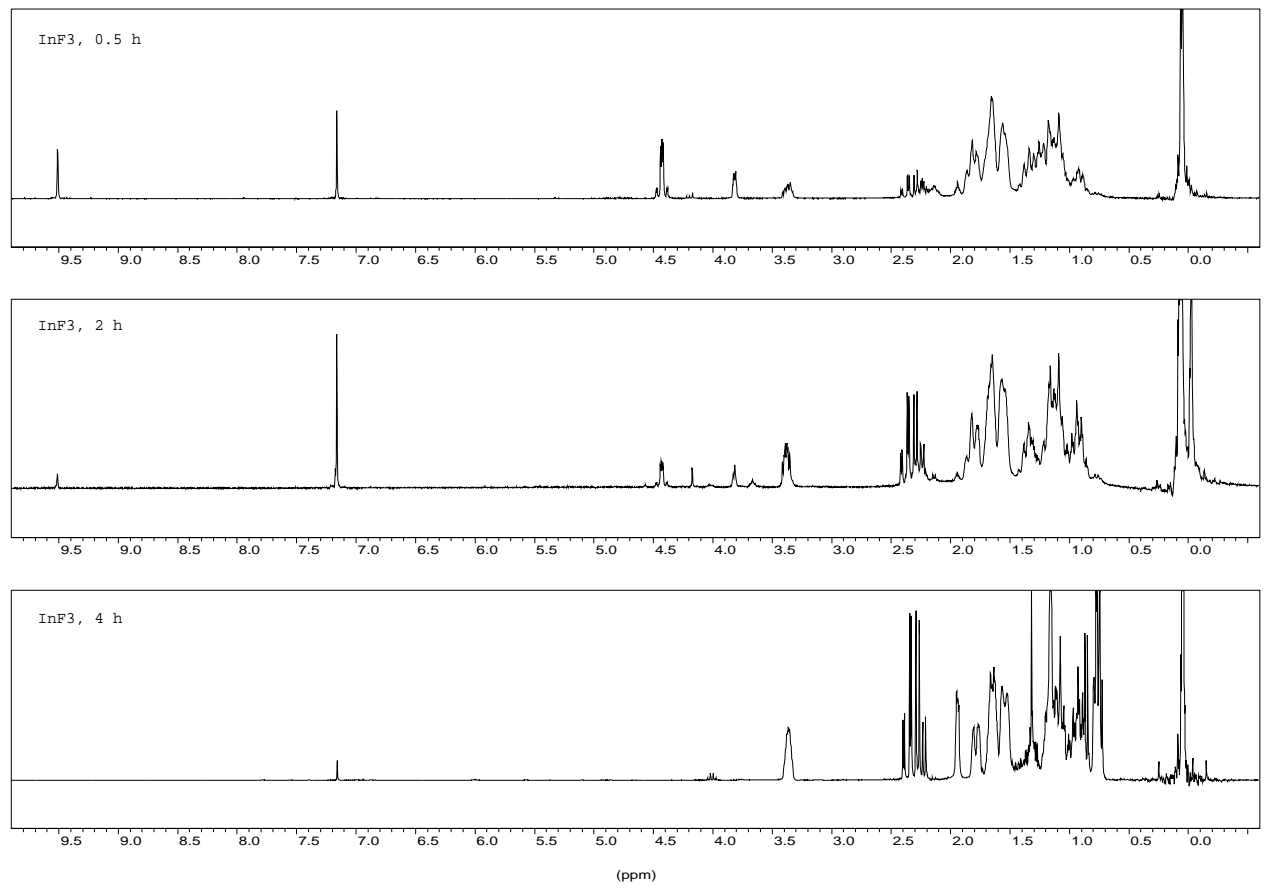


\section{References}

1. Isaac, M. B.; Chan, T. H. Chem. Commun. 1995, 1003.

2. Hammond, G. B.; Wang, Z. G. J. Org. Chem. 2000, 65, 6547.

3. Loh T. P.; Hu, Q. Y.; Chok, Y. K.; Tan, K. T. Tetrahedron Lett. 2001, 42, 9277. 